\title{
Indigestible mitochondria cause heartburn
}

\author{
Roberta A Gottlieb ${ }^{1}$, Phyllis-Jean Linton ${ }^{1}$ \\ ${ }^{1}$ Donald P. Shiley BioScience Center, San Diego State University, San Diego, CA 92182, USA \\ Cell Research (2012) 22:1518-1520. doi:10.1038/cr.2012.96; published online 19 June 2012
}

The link between impaired autophagic flux (autophagus interruptus), damaged mitochondria, and myocardial inflammation has been further tightened with the recent paper by Oka and colleagues, in which failure to degrade mitochondrial DNA exacerbated myocardial inflammation in the context of pressure overload. Using mice with cardiac-specific deletion of the lysosomal DNase II that were subjected to aortic banding, Otsu's group showed that mitochondrial DNA accumulated in lysosomes and resulted in TLR9dependent production of inflammatory cytokines.

The link between autophagy, mitochondria, and inflammation in the heart has been further tightened with the recent paper in Nature by Oka et al [1]. In this work, Otsu's team showed that mitochondrial DNA (mtDNA) that escapes autophagy led to the activation of TLR9 and inflammation. Mice with cardiac-specific deletion of lysosomal DNase II (DNase2a $a^{--}$) developed accelerated myocarditis and heart failure after the induction of pressure overload with thoracic transverse aortic constriction (TAC). Cardiomyocytes in TAC-treated DNase $2 a^{--}$mice showed increased expression of IL-1 $\beta$ and IL-6, which was followed by infiltration of $\mathrm{CD}^{+} 8^{+}$macrophages and $\mathrm{Ly}_{6 \mathrm{G}}{ }^{+}$cells.

Correspondence: Roberta A Gottlieb

E-mail: robbieg@sciences.sdsu.edu
Mitochondrial DNA was observed in autolysosomes in the hearts of these mice, indicating that mitochondria were engulfed into autophagosomes that subsequently fused with lysosomes; however, because of the absence of DNase II, mtDNA could not be degraded. They hypothesized that mtDNA, which resembles bacterial (due to unmethylated CpG motifs), would activate TLR9, which is present in endolysosomes and could potentially interact with undigested mtDNA in autolysosomes. Using inhibitory oligodeoxynucleotides or mice with Tlr9 deletion, Oka et al. showed that TLR9 was indeed responsible for the inflammatory signaling, leading to myocarditis and failure after TAC.

Depolarized or damaged mitochondria are recognized and targeted for removal via PINK1/Parkin and p62, which recruits the forming autophagosome to the ubiquitin-decorated mitochondrial fragment. Ubiquitination of mitofusin 2 (Mfn2) prevents fusion with the remainder of the mitochondrial network [2], and participation of Drp1 and Mfn2 in mitophagy has been established [3, 4]. Nix and Bnip3 have been shown to stimulate mitophagy, likely through triggering depolarization and subsequent Parkin recruitment [5]. A maladaptive role for Nix and Bnip3 has been demonstrated, which might lead one to suspect that autophagy/ mitophagy is deleterious. However, Otsu's group previously showed that inactivation of autophagy exacerbated remodeling after TAC [6], although Hill's group, using Beclin $1^{+/-}$mice, reached a different conclusion [7]. Further work has revealed that intact autophagic flux is adaptive [8], but in settings where flux is impaired, as in the DNase $2 a^{-/-}$mice described in the paper by Oka et al. [1], impaired flux is deleterious. While the authors suggest that mtDNA released from lysosomes is responsible for the inflammatory signaling, it is possible that the overall impaired mitochondriato-lysosome flux results in a backup of damaged mitochondria that release their contents to the cytoplasm before they can be sequestered in autophagosomes. The pathogenic consequences of impaired autophagy and coined autophagus interruptus have been a focus of recent interest [9].

Pattern recognition receptors (PRRs) are comprised of Toll-like receptors (TLRs), nucleotide-binding oligomerization domain (NOD)-like receptors (NLRs) and retinoic acid induciblegene-1 (RIG)-like receptors (RLRs) and were initially shown to regulate antimicrobial host defense through their recognition of microbial molecular motifs called pathogen-associated molecular patterns (PAMPs). Since their discovery, PRRs have been shown to have many physiological functions, including their ability to sense endogenous molecules or danger signals that are released in injured tissues and to maintain tissue homeostasis by regulating tissue repair and regeneration. Growing evidence has demonstrated that TLRs play a critical role in myocardial ischemia/reperfusion 
(I/R) injury. Specifically, a deficiency of TLR4 protects the myocardium from ischemic injury whereas modulation of TLR2 induces cardioprotection against ischemic insult $[10,11]$. Recent work suggests a role for NLR protein, Nlrp3, in the inflammation- and cardiac injury-induced post ischemia. PRRs are expressed in a variety of cell types and the binding to TLRs by PAMPs triggers the release of proinflammatory mediators [12]. Numerous studies have shown that $\mathrm{I} / \mathrm{R}$ significantly increases the gene expression of the inflammatory cytokines, IL-1 $\beta$, IL- 6 and TNF $\alpha$, in the myocardium. These proinflammatory cytokines appear to be directly involved in the progression of cardiac injury, myocardial dysfunction, vascular wall remodeling, heart failure and hypertrophy. In cardiac I/R injury, studies with TLR2- and TLR4-deficient mice have suggested a crucial involvement of these receptors in the early process of inflammation. Based on in vitro studies, many TLR ligands, such as heat shock proteins, high-mobility group box 1 protein, ATP, hyaluronan, and heparin sulfate, have been shown to initiate sterile inflammation [13]. However, there is still no direct evidence in vivo to demonstrate that these molecules are the drivers of the inflammatory response and the pathogenesis of heart failure. Moreover, the mechanisms responsible for the initiation and coordination of the inflammation in the heart are less understood.

Oka et al. [1] present a compelling story in which mtDNA, a byproduct of insufficient mitophagy, induces sterile inflammation and subsequent cardiac dysfunction after TAC through the TLR9 pathway. The data not only identifies a new endogenous 'danger' molecule and its PRR pathway but their study also alludes to insufficient mitophagy as initiating the response. This study confirms the close inter-relationship between autophagy and members of the PRR family in mediating inflammation. This relationship has been intensively studied with the NLRP3 inflammasome [14]. In these studies, blocking autophagy potentiated NLRP3 inflammasome activity, whereas stimulating autophagy induced inflammasome recruitment to the autophagosome for degradation. Moreover, the induction of inflammation by the NLRP3 inflammasome has been demonstrated as a mechanism underlying many chronic sterile inflammatory-related diseases, such as gout and type II diabetes mellitus [15]. Future studies with inflammation and TLR9 are likely to follow in the footsteps of the NLRP3 inflammasome. They are likely to include studies that increase our understanding of the pathways in which autophagy and TLR9 activation by mtDNA might regulate each other, and whether the mitophagy-TLR9-inflammation axis as described by Oka et al. is a general mechanism applicable to many chronic inflammatory diseases.

While DNase2a deficiency is not a common human condition, impaired autophagic flux is far more widespread. Increasing data from animal studies point to aging and metabolic syndrome as two important settings in which autophagic flux is impaired. If impaired flux as well as inefficient lysosomal degradation can lead to mtDNA interaction with TLR9, then it is likely that these conditions will result in increased inflammatory signaling. It is already well established that aging and metabolic syndrome are accompanied by increased inflammation, giving rise to the terms "inflammaging" and "meta-flammation". It will be important to determine whether impaired mitophagy and/or inefficient lysosomal degradation contribute to the inflammatory state associated with these conditions. Furthermore, it will be equally important to determine the pathways by which inflammation and mitophagy are linked, whether other damaged organelles can also activate inflammation through PRRs, and whether this process is relevant to stress in other organs.

\section{References}

1 Oka T, Hikoso S, Yamaguchi O, et al. Mitochondrial DNA that escapes from autophagy causes inflammation and heart failure. Nature 2012; $\mathbf{4 8 5}$ :251255.

2 Poole AC, Thomas RE, Yu S, Vincow ES, Pallanck L. The mitochondrial fusion-promoting factor mitofusin is a substrate of the PINK1/parkin pathway. PLoS One 2010; 5:e10054.

3 Zhao T, Huang X, Han L, et al. Central role of mitofusin 2 in autophagosomelysosome fusion in cardiomyocytes. $J$ Biol Chem 2012; 287:23615-23625.

4 Gomes LC, Di Benedetto G, Scorrano L. During autophagy mitochondria elongate, are spared from degradation and sustain cell viability. Nat Cell Biol 2011; 13:589-598.

5 Lee Y, Lee HY, Hanna RA, Gustafsson ÅB. Mitochondrial autophagy by Bnip3 involves Drp1-mediated mitochondrial fission and recruitment of Parkin in cardiac myocytes. Am J Physiol Heart Circ Physiol 2011; 301:H1924-H1931.

6 Nakai A, Yamaguchi O, Takeda T, et al. The role of autophagy in cardiomyocytes in the basal state and in response to hemodynamic stress. Nat Med 2007; 13:619-624.

7 Zhu H, Tannous P, Johnstone JL, et al. Cardiac autophagy is a maladaptive response to hemodynamic stress. J Clin Invest 2007; 117:1782-1793.

8 Huang C, Andres AM, Ratliff EP, Hernandez G, Lee P, Gottlieb RA. Preconditioning involves selective mitophagy mediated by Parkin and p62/ SQSTM1. PLoS One 2011; 6:e20975.

9 Gottlieb RA, Mentzer RM. Autophagy during cardiac stress: Joys and frustrations of autophagy. Annu Rev Physiol 2010; 72:45-59.

10 Favre J, Musette P, Douin-Echinard $\mathrm{V}$, et al. Toll-like receptors 2-deficient mice are protected against postischemic coronary endothelial dysfunction. Arterioscler Thromb Vasc Biol 2007; 27:1064-1071.

11 Fallach R, Shainberg A, Avlas O, et al. Cardiomyocyte toll-like receptor 4 is involved in heart dysfunction following septic shock or myocardial ischemia. $J$ Mol Cell Cardiol 2010; 48:1236-1244.

12 Mezzaroma E, Toldo S, Farkas D, et al. The inflammasome promotes adverse cardiac remodeling follow- 
ing acute myocardial infarction in the mouse. Proc Natl Acad Sci USA 2011; 108:19725-19730.

13 Chen GY, Nuñez G. Sterile inflammation: sensing and reacting to damage. Nat Rev Immunol 2010; 10:826-837.
14 Shi CS, Shenderov K, Huang NN, et al. Activation of autophagy by inflammatory signals limits IL-1 production by targeting ubiquitinated inflammasomes for destruction. Nat Immunol 2012; 13:255-263.
15 Schroder K, Zhou R, Tschopp J. The NLRP3 inflammasome: a sensor for metabolic danger. Science 2010; 327:296-300. 